\title{
Evaluation of a Bearingless PM Motor with Wide Magnetic Gaps
}

\author{
Junichi Asama, Member, IEEE, Miya Amada, Norimasa Tanabe, Naoya Miyamoto, \\ Akira Chiba, Fellow, IEEE, Satoru Iwasaki, Masatsugu Takemoto, Member, IEEE, \\ Tadashi Fukao, Fellow, IEEE, and M. Azizur Rahman, Life Fellow, IEEE
}

\begin{abstract}
A new non-contact drive system has been developed employing a consequent-pole type bearingless permanent magnet (PM) motor and a thrust magnetic bearing. This bearingless motor has a wide magnetic gap. The wide magnetic gap may cause a decrease in radial magnetic suspension force because of an increase in magnetic reluctance. A thrust magnetic bearing is installed at the end of the shaft, because strong thrust force exists in centrifugal pump applications. In this paper, the magnetic suspension forces and rotation characteristics of the experimental prototype are evaluated, while the rotor is magnetically suspended. Experimental results are also compared to the calculated ones using the finite element method. It is demonstrated that the developed consequent-pole type bearingless PM motor possesses sufficient magnetic suspension force for non-contact operation.
\end{abstract}

Index Terms-Bearingless motor, consequent-pole, magnetic bearing, PM motor, centrifugal pump, wide gap.

\section{INTRODUCTION}

A BEARINGLESS motor combines functions of both noncontact magnetic suspension and torque generation together in a single motor [1]. Magnetic suspension offers the advantage of no wear particles, less maintenance, and high rotational speed as compared to mechanical suspension that causes mechanical friction. In addition, a bearingless motor possesses the advantages of a simple structure, compactness, and cost reduction, in comparison to a conventional tandem structure consisting of magnetic bearings and a motor.

Recently, bearingless motors are practically applied to centrifugal pumps in industries where an infusion of wear particles in pumping medium should be avoided [2]-[5]. In such pumps, protective covers must be attached to both rotor and stator surfaces in order to prevent both medium contamination and material corrosion. These covers should be as thick as possible to enhance mechanical robustness and reliability. It is necessary to design such a motor with wide

J. Asama is with the Department of Mechanical Engineering, Shizuoka University, Hamamatsu 432-8561, Japan (email: tjasama@ipc.shizuoka.ac.jp)

M. Amada, N. Tanabe, N. Miyamoto, A. Chiba, and T. Fukao are with the Department of Electrical Engineering, Tokyo University of Science, Chiba 278-8510, Japan (e-mail:chiba@rs.noda.tus.ac.jp).

S. Iwasaki is with the Department of Mechanical Systems Engineering, Musashi Institute of Technology, Tokyo 158-8557, Japan.

M. Takemoto is with the Department of Information Science and Technology, Hokkaido University, Hokkaido 060-0808, Japan.

M. A. Rahman is with the Discipline of Engineering and Applied Science, Memorial University of Newfoundland, Newfoundland A1B 3X5, Canada. magnetic gap between a rotor and a stator. These covers and the medium are not generally made of ferromagnetic material, thus magnetic reluctance is as high as the air. In conventional motors, the gap factor $g / r$ is designed to be from about 0.005 to 0.02 , where $g$ and $r$ are the magnetic gap and rotor radius, respectively. The authors consider that the wide gap can be defined as the condition when the gap factor $g / r$ is larger than 0.1 .

In bearingless motors [6]-[8] and magnetic bearings [9][10] the magnetic gaps are generally designed to be relatively narrow. A wide magnetic gap causes a decrease in magnetic suspension force because of an increase in reluctance. A serious condition occurs at suspension start-up from touched down condition. In this condition strong magnetic attractive force is generated by rotor permanent magnets. Active magnetic force is required to overcome the attractive force to lift a shaft.

A two-axis bearingless centrifugal pump is reported in reference [11]. In this bearingless pump, two radial positions are actively controlled, while the thrust and tilting are passively regulated. This structure provides the advantage of simple and low cost. However, rather large clearance is required for a propeller of the pump. The clearance reduces the overall pump efficiency.

In this paper, a novel 5-axis controlled non-contact bearingless drive system has been developed with wide magnetic gaps. The prototype machine mainly consists of two consequent-pole type permanent magnet (PM) bearingless motor units [6] and a thrust magnetic bearing. The magnetic gaps are much wider than those of conventional bearingless machines. The gap factor is more than 0.13 . The aim of this project is the realization of a novel centrifugal pump with bearingless motors. The objective of this paper is the evaluation and the assessment of the magnetic suspension and motor characteristics. The evaluation test is composed of (a) magnetic suspension start-up, (b) static radial force, (c) radial and thrust interference, (d) static thrust force, (e) static torque, and $(\mathrm{f})$ rotation and vibration.

A part of this paper was presented in the Panel Session, sponsored by Motor Sub-Committee in 2008 IEEE-PES General Meeting [12]. Evaluation tests (a) and (b) in the preceding paragraph have been presented. Significant improvements with additional tests (c), (d), (e) and (f) listed in the preceding paragraph are included in this paper. 


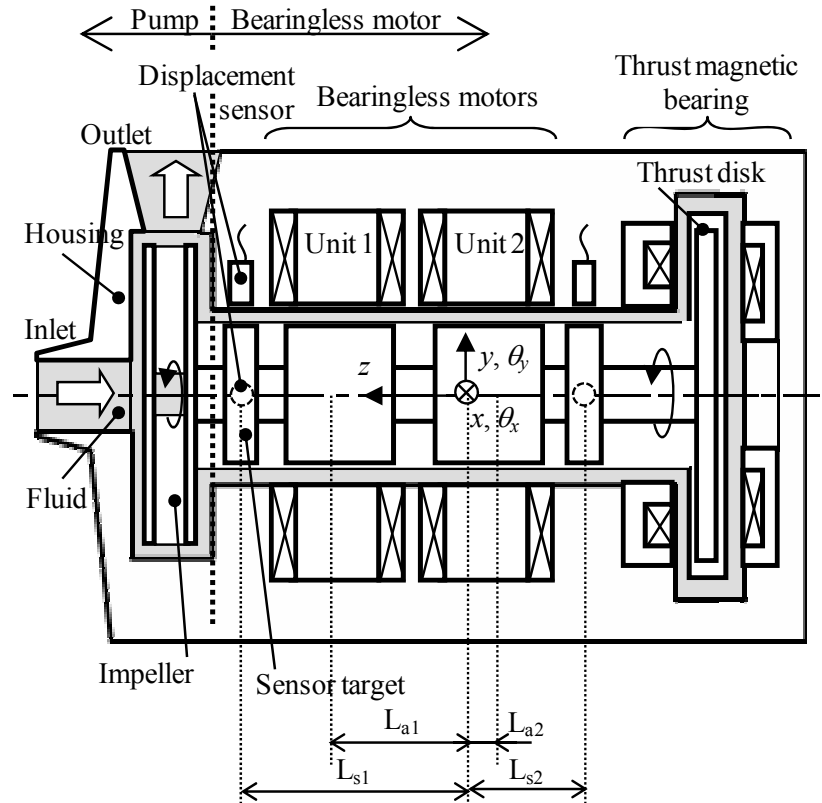

Fig. 1. A schematic of a centrifugal pump with bearingless drive.

\section{WIDE GAP BEARINGLESS DRIVE SYSTEM}

\section{A. Structure of Bearingless Motor Pump}

Fig. 1 shows a schematic of the proposed bearingless centrifugal pump and the coordinate definition. The coordinate origin is gravity center of the rotor shaft. $x, y$ and $z$ are translational motion of the rotor. $\theta_{x}$ and $\theta_{y}$ are the tilting motion. Two bearingless motor units are connected in tandem. A thrust magnetic bearing is installed on the right hand side of the shaft. It consists of a thrust disk and two annular coils. On the left hand side, a pump impeller is attached. The bearingless motor can actively regulate both radial $(x, y)$ and tilting $\left(\theta_{x}, \theta_{y}\right)$ motions of the rotor. The thrust position $(z)$ is actively controlled by the thrust magnetic bearing. Thus five eddy current displacement sensors are installed. The rotor can be magnetically suspended and rotated without mechanical contact in radial and thrust directions. Pumping medium is sealed with protective covers attached to both the rotor outside surface and the stator inner surface. It should be noted that magnetic gaps are 4-5 mm, much wider than the conventional bearingless machines.

\section{B. Principle of Suspension Force Generation}

The bearingless motors in the centrifugal pump are consequent-pole types. Fig. 2 shows a consequent-pole type bearingless PM motor. Radially magnetized PMs are inset between the rotor iron poles. The bias fluxes are generated by $\mathrm{PMs}$ so that the iron poles are magnetized as N-poles. The rotor iron between the PMs is consequently magnetized. Therefore, this consequent-pole type bearingless PM rotor has eight poles.

Fig. 2 also shows principles of the radial force generation when the rotor angle $\theta_{z}$ is $0^{\circ}$. The suspension magnetic flux shown in Fig. 2 is generated by the current in the suspension coil. The airgap flux densities in the right and left directions are increased and decreased, respectively. The flux density at the magnetic gaps are unbalanced because of superposition of the suspension flux on the PM bias fluxes. As a result, suspension force is generated in the positive $x$-direction.

The negative $x$-direction force can be generated by providing negative current in suspension winding. The $y$ direction force can be generated by currents in a suspension winding perpendicular to the excited suspension winding. The total suspension force is generated by a vector sum of the $x$ and $y$-direction forces. Even though the rotor is rotated, the same suspension winding current can be provided. The suspension radial force is independent of the rotor rotational angular position [6]. This is one of the advantages of the consequent-pole type bearingless motors. This radial suspension force is proportional to the suspension current.

\section{Control System}

In the radial directions, centralized position regulations are installed. Fig. 3 shows the control systems in $x$ - and $\theta_{y^{-}}$ directions. The control systems in $y$ - and $\theta_{x}$ - directions are identical. The $x_{1}$, and $x_{2}$ are output signals from displacement sensors of unit 1 and unit 2, respectively. The $x$ and $\theta_{y}$ are rotor positions in the radial and tilting directions, respectively. The $x^{*}$ and $\theta_{y}{ }^{*}$ indicate position references in the control system. The $i_{x}{ }^{*}$ and $i_{\theta y}{ }^{*}$ are calculated current commands in radial and tilting directions, respectively. The $i_{a 1}{ }^{*}$ and $i_{a 2}{ }^{*}$ are also current commands in the $x$ direction in unit 1 and unit 2, respectively. Control boxes INV and RD indicate abbreviations of the inverter and rotor dynamics, respectively. The input current to the RD block induces suspension force on the rotor. Due to the rotor dynamics, the rotor displaces in the radial direction. The output of the RD block is the radial displacement of the rotor.

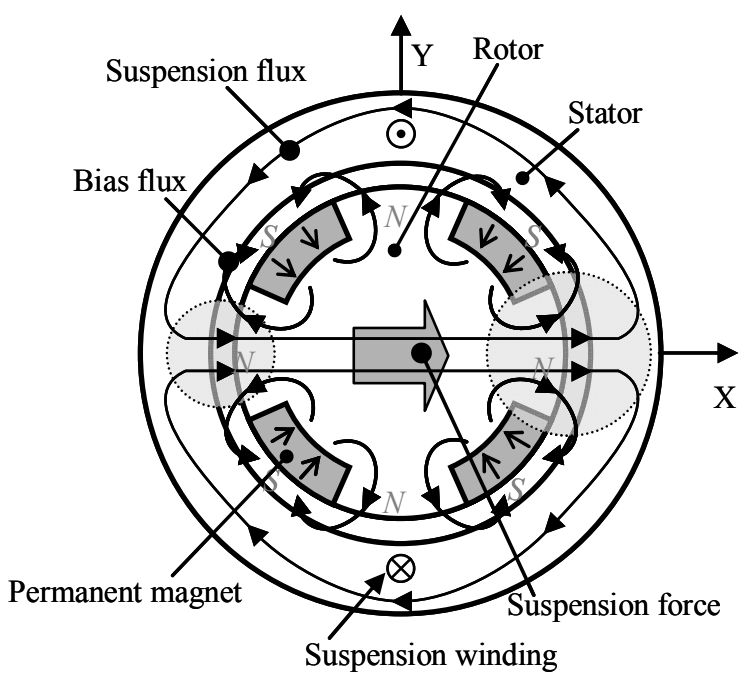

Fig. 2. A structure and a principle of suspension force generation in a consequent-pole bearingless PM motor. 


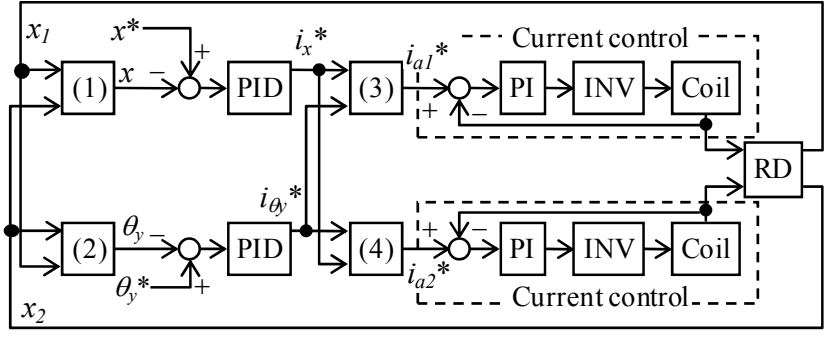

Fig. 3. Control system in $x$ - and $\theta_{y}$ - directions.

The sensor outputs are transformed into the movements of the center of the gravity, i.e., parallel and rotational movements. From the sensor outputs $\left(x_{1}, x_{2}\right), x$ and $\theta_{y}$ are calculated by following equations:

$$
\begin{aligned}
& x=\frac{L_{s 2} x_{1}+L_{s 1} x_{2}}{L_{s 1}+L_{s 2}} \\
& \theta_{y}=\frac{x_{1}-x_{2}}{L_{s 1}+L_{s 2}}
\end{aligned}
$$

where, $L_{\mathrm{s} 1}$ and $L_{\mathrm{s} 2}$ are the distances from the shaft gravity center to each sensor as previously shown in Fig.1. The control boxes (1) and (2) indicate these calculations.

The parallel and rotational movements are compared and these errors are amplified by the proportional-integralderivative (PID) controllers. As a result, the current commands $i_{x}{ }^{*}$ and $i_{\theta y}{ }^{*}$ are calculated. From the $i_{x}{ }^{*}$ and $i_{\theta y}{ }^{*}$, suspension current commands $i_{a 1}{ }^{*}$ and $i_{a 2}{ }^{*}$ are calculated by following equations, as,

$$
\begin{aligned}
& i_{a 1}{ }^{*}=\frac{2 L_{a 2}}{L_{a 1}+L_{a 2}} i_{x}{ }^{*}+i_{\theta_{y}}{ }^{*} \\
& i_{a 2}{ }^{*}=\frac{2 L_{a 1}}{L_{a 1}+L_{a 2}} i_{x}{ }^{*}-i_{\theta_{y}}{ }^{*}
\end{aligned}
$$

where, $L_{\mathrm{a} 1}$ and $L_{\mathrm{a} 2}$ are the distances from the shaft gravity center to each unit center as previously shown in Fig. 1. The control boxes (3) and (4) indicate these current command calculations. A proportional-integral (PI) loop is constructed so that the suspension currents of $i_{a 1}$ and $i_{a 2}$ follow the current references of $i_{a 1}{ }^{*}$ and $i_{a 2}{ }^{*}$, respectively.

In thrust direction, a disk and two coils are installed. The coil of bearingless motor side is $u$-phase. The other coil is $w$ phase. Fig. 4 shows the control system in $z$-direction. This control system is almost the same as the control system in radial directions. The sensor output $(z)$ is compared with the reference $z^{*}$ and the error is amplified by the PID controller. The current command $i_{z}{ }^{*}$ is divided into $i_{z u}{ }^{*}$ and $i_{z w}{ }^{*}$ by following equations, as,

$$
\begin{aligned}
& i_{z u}{ }^{*}=-\left(i_{z u b}+i_{z}{ }^{*}\right) \\
& i_{z w}{ }^{*}=i_{z w b}-i_{z}{ }^{*}
\end{aligned}
$$

where, the $i_{z u b}$ and $i_{z w b}$ are bias currents of this magnetic bearing. To realize the linear relationships between the suspension force and current, the bias currents are provided in this magnetic bearing. The control box (5), (6) of Fig. 4 indicates the calculation of the above equations.

\section{Target Specification}

Table 1 summarizes specification required in bearingless motor system. The target specification is set by the authors' group. A motor torque of $1.9 \mathrm{Nm}$ is needed, which is equal to a motor output of $1.1 \mathrm{~kW}$ at a rotational speed of 5,500 r/min.

\section{SUSPENSION CHARACTERISTICS}

\section{A. Test System}

Figure 5 shows a test machine of the 5-axis controlled bearingless motor. An impeller and a pump housing are not installed in the test machine. Table II shows specifications required in the bearingless PM motor. The frame diameter and length are $186 \mathrm{~mm}$ and $182 \mathrm{~mm}$, respectively. The length includes the sensor targets, bearingless motors and the thrust magnetic bearing. The rotor diameter is $64 \mathrm{~mm}$. The magnetic gap of 4-5 mm includes protective covers and mechanical gaps. Commercially available eddy current displacement sensors are used in order to detect the radial position of the

TABLE I

DESIGN GOALs OF BEARINGLESS PM MOTOR SYSTEM

\begin{tabular}{ll}
\hline Static radial suspension force & $100 \mathrm{~N}$ \\
\hline Static thrust suspension force & $110 \mathrm{~N}$ \\
\hline Motor output & $1.1 \mathrm{~kW}$ \\
\hline Rotational speed & $5,500 \mathrm{r} / \mathrm{min}$ \\
\hline
\end{tabular}

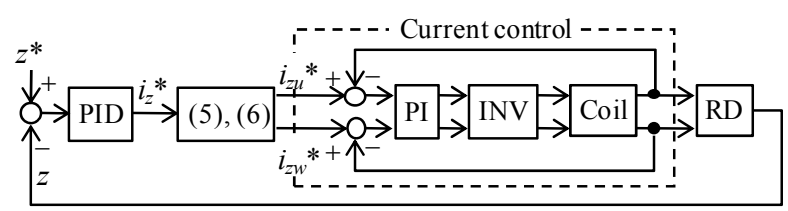

Fig. 4. Control system in $z$-direction.

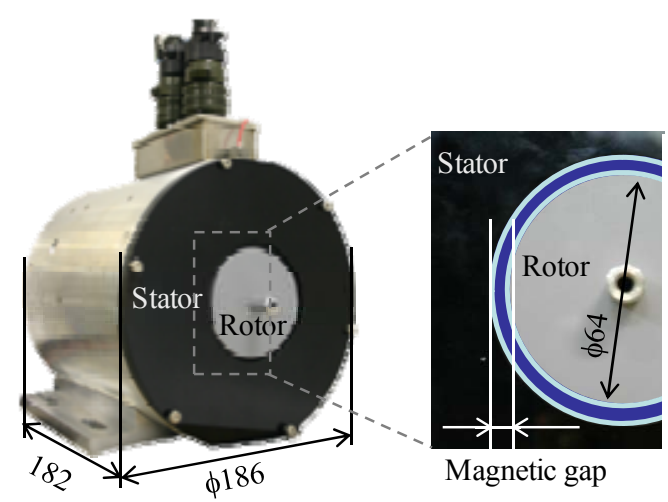

Fig. 5. A test machine of the developed bearingless PM motor. 
TABLE II

SPECIFICATIONS OF BEARINGLESS PM MOTOR

\begin{tabular}{ll}
\hline Outer diameter of motor & $186 \mathrm{~mm}$ \\
\hline Axial length of motor & $182 \mathrm{~mm}$ \\
\hline Motor voltage & $200 \mathrm{~V}$ \\
\hline Motor current & $8 \mathrm{~A}$ \\
\hline
\end{tabular}

rotor.

The test machine is driven by $200 \mathrm{~V}$ three-phase, voltage source IGBT inverters with DC bus voltage of $280 \mathrm{~V}$. A series number of turns for one-phase suspension windings is 155 . The motor windings are connected to the PWM inverter. The blocks shown in Fig. 3 and Fig. 4 are realized using a commercial microprocessor. The switching frequency of the inverter is $20 \mathrm{kHz}$. The microprocessor and its sampling rate for magnetic suspension control are SH7047 (Renesas Technology Corp., Japan) and $300 \mu$ s, respectively. In the following, the experiments are carried out in the air.

\section{B. Radial Start-up}

First, radial start-up of the consequent-pole type bearingless PM motor is experimentally investigated. The rotor is actively regulated with a 4 -axis $\left(x, y, \theta_{x}, \theta_{y}\right)$ active controller. The thrust magnetic bearing is not operated. Fig. 6(a) shows radial and tilting positions at start-up. Fig. 6(b) shows suspension winding current waveforms.

In Fig. 6(a), before the controller is switched on, the rotor is touched down in the negative $y$-direction because of the gravity. When the suspension controller is switched on, the rotor moves to certain positions. In order to reduce the suspension currents, all position references are adjusted as $x^{*}=0.2 \mathrm{~mm}, y^{*}=0.4 \mathrm{~mm}, \theta_{x}{ }^{*}=0 \mathrm{mrad}$, and $\theta_{y}{ }^{*}=3 \mathrm{mrad}$. The position references are given so that unbalanced magnetic pull force is mostly balanced with the rotor shaft weight.

In Fig. 6(b), $i_{a 1} i_{b 1}, i_{a 2}$ and $i_{b 2}$ are suspension winding currents of a- and b-phase in the unit 1 and 2, respectively. The steady-state suspension currents are less than $1 \mathrm{~A}$. The current values are intentionally reduced by adjusted reference positions. Figures 6(a) and 6(b) show that this test machine can be magnetically suspended. The performance requirement of radial suspension start-up from power off condition is satisfied.

\section{Radial Suspension Force}

The static radial force and current characteristics are measured. External forces are applied at both ends of the shaft, then radial suspension force is measured. There are currents on $y$-direction because of the gravity. Therefore, this experiment is executed in the $x$-direction.

Fig. 7(a) shows the experimental setup. External forces are applied at both shaft ends of unit 1 and unit 2 . The thrust magnetic bearing is switched off and shaft extension is attached to the end of the thrust disk. The center of gravity is not at the center of the shaft due to weight of thrust disk, as shown in Fig. 1. Consequently, if the same external force is
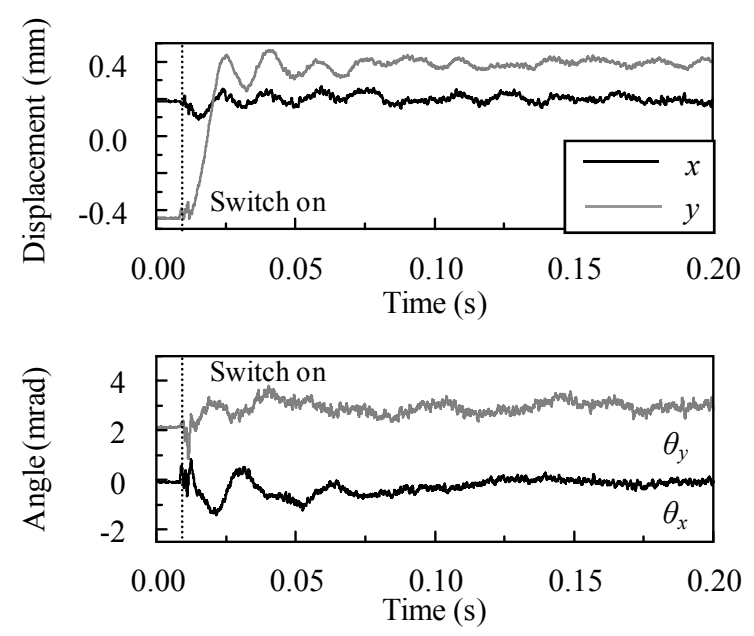

(a) Rotor positions in radial and tilting directions.

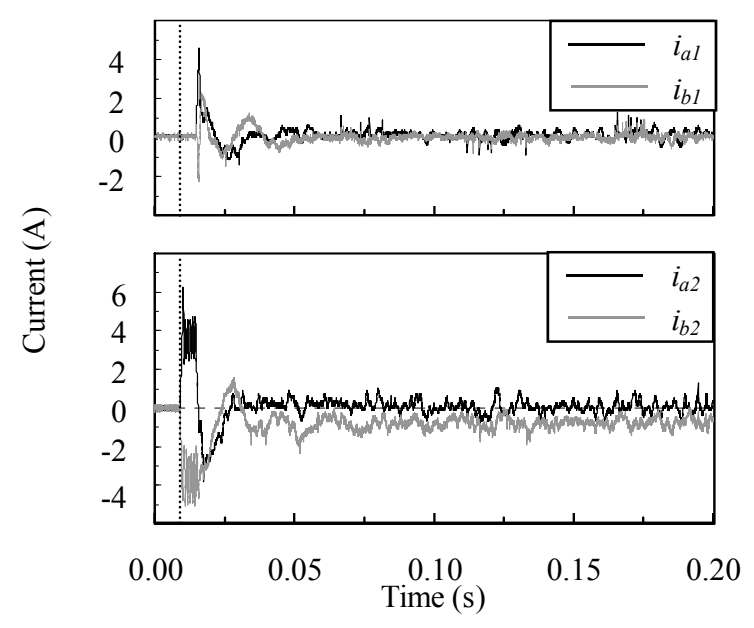

(b) Currents in suspension windings of the bearingless motor.

Fig. 6. Waveforms of radial positions and suspension currents of the bearingless PM motor at start-up without an operation of a thrust magnetic bearing.

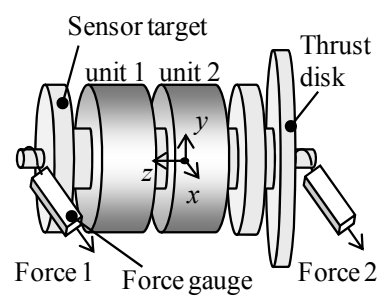

(a) An experimental setup and method for an application of external forces Force 1 and 2.

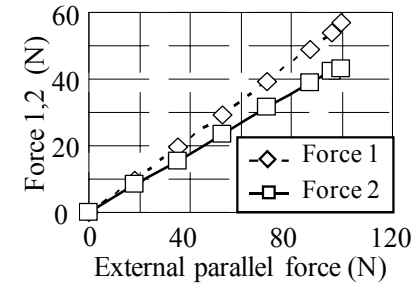

(b) Relationships between the external force and Force 1 and 2 .
Fig. 7. Experimental method for measuring radial suspension force of the bearingless PM motor. 


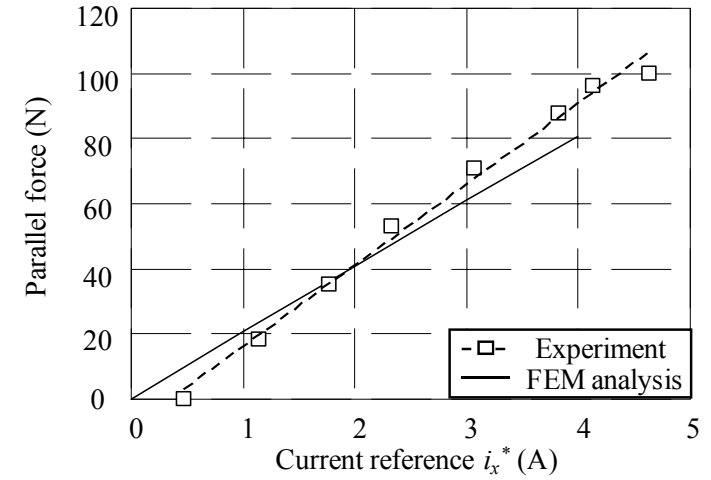

Fig. 8. Measured and calculated parallel force of the bearingless PM motor against suspension current when external forces are applied.

applied to both ends, a moment is resulted in the tilting direction. To eliminate such tilting moment, and to apply only parallel external force, the applied forces are adjusted at each shaft end.

The applied forces in this experiment are shown in Fig. 7(b). The applied forces of unit 1 and unit 2 are Force 1 and Force 2, respectively. These forces are adjusted so that there is no moment in $\theta_{y}$-direction. The sum of the Force 1 and Force 2 is the applied external parallel force $F$.

Fig. 8 shows the applied external parallel force against the suspension current reference $i_{x}{ }^{*}$ generated in the controller. It is noted that parallel suspension force of $100 \mathrm{~N}$ is applied. The experimental results are compared to the calculated ones provided by the Finite Element Method (FEM). Commercially available three-dimensional FEM software package (JMAGStudio, JSOL Corp., Japan) is used. The data points are subjected to least-squares fitting. In Fig. 8, the solid and broken lines indicate the FEM simulation and experimental results, respectively. A suspension force constant is defined as force by current. The measured and calculated suspension force constants are $24.9 \mathrm{~N} / \mathrm{A}$ and $22.6 \mathrm{~N} / \mathrm{A}$, respectively. There is an error of $4.8 \%$.

In this machine, the rated current of the suspension winding is $4.5 \mathrm{~A}$. With the rated current, the suspension force is $100 \mathrm{~N}$. The performance requirement of static radial suspension force of $100 \mathrm{~N}$ is satisfied. The radial levitation current is not zero with zero applied force because the center of the mechanical structure does not coincide with the magnetic center of the bearingless motor.

\section{Thrust Start-up}

When the rotor is actively regulated with a 4-axis active controller, the passive force exists in thrust direction. Thus, thrust direction is passively stable. However, strong thrust force occurs in pump operation. Hence, the thrust magnetic bearing has been employed in thrust direction.

Fig. 9 shows the thrust and radial positions and currents in response to an impulse force in the thrust direction. The upper waveforms show the thrust displacement $z$ and the currents $i_{\mathrm{zu}}$ and $i_{\mathrm{zw}}$ of the thrust magnetic bearing. The lower waveforms

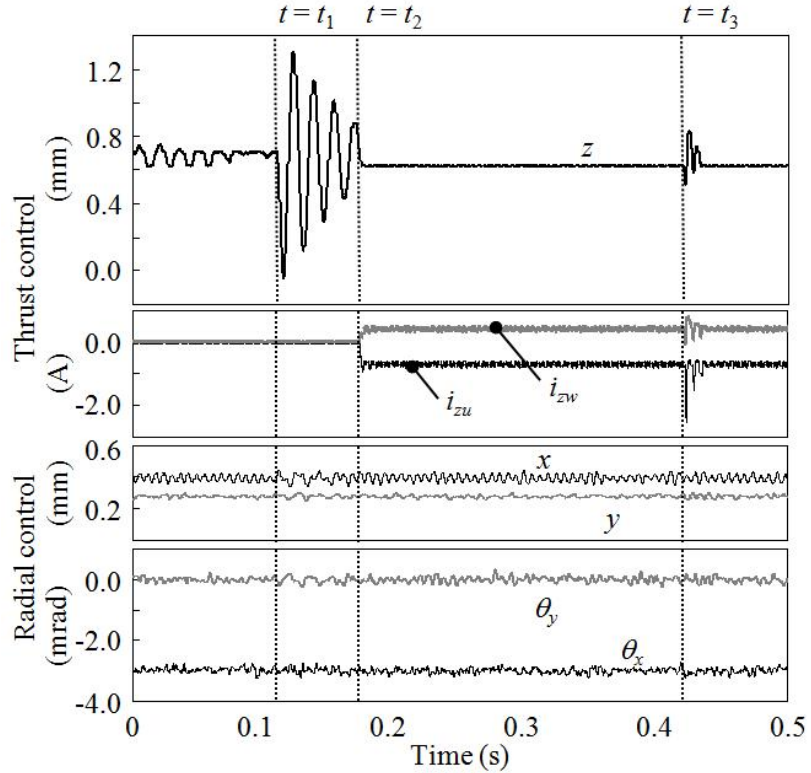

Fig. 9. Thrust, radial and tilting positions and currents of thrust magnetic bearing in response to impulse external force in thrust direction.

show the radial displacements and angles.

At $t=0$, the rotor is suspended by the bearingless motor in radial and tilting directions. The thrust motion is stable due to the effect of passive stabilization of bearingless motor. At $t=t_{1}$, an external impulsive force is applied, and then the rotor has thrust vibration. Although the vibration is gradually converged due to the thrust passive stabilization, the damping effect is not sufficient.

At $t=t_{2}$, the thrust active control is switched on, then, the vibration is suppressed by active thrust magnetic force. The thrust position is regulated at about $0.6 \mathrm{~mm}$. It is almost magnetic center position of the bearingless motor. The $u$ phase and $w$-phase bias current is $1.5 \mathrm{~A}$ and $1 \mathrm{~A}$, respectively. The stator structures of the thrust magnetic bearing are different as shown in Fig. 1. Therefore, suspension force constants are different. The difference of suspension force constants causes the difference of the bias current.

In the radial position waveforms before and after $t=t_{2}$, the vibration amplitude is slightly changed. This change shows the interaction between the thrust and radial suspension. The thrust movement causes a change in the reluctance, because the rotor and stator iron are misaligned.

At $t=t_{3}$, an external impulsive force is applied, again. The rotor is almost kept in regulated position by the thrust magnetic suspension currents. This figure shows that this machine can start up and tolerate the thrust direction disturbance.

\section{E. Thrust Suspension Force}

In this sub-section, static thrust force and current characteristics are measured. In 5-axis actively controlled condition, external thrust force is applied at the shaft end. The direction of the thrust force is in the positive $z$ direction. In the 


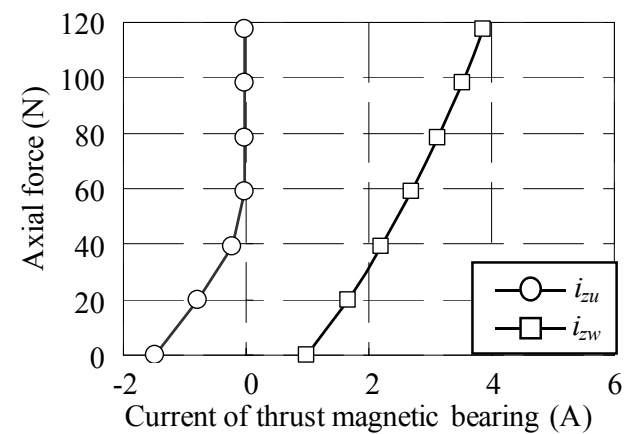

Fig. 10. Applied axial force against currents of the thrust magnetic bearing in 5-axis magnetic suspension.

pump operation, the positive thrust force should be generated to balance to the pump impeller force. Fig. 10 shows the applied force against the currents of the thrust magnetic bearing. Within the rated current of $4.5 \mathrm{~A}$, this machine can generate $120 \mathrm{~N}$, which is higher than the thrust force requirement of $110 \mathrm{~N}$. This experimental results show that the target thrust suspension force is satisfied.

\section{TORQUE CHARACTERISTICS}

In this section, the static torque characteristic is measured. The test machine is mechanically connected to a dc machine through a torque transducer. The rotational angular position is mechanically fixed. The rated current of $8 \mathrm{~A}$ is supplied in eight-pole three-phase motor windings. A q-axis current of 13.8 A is supplied to the motor windings. The static torque is then measured with the torque transducer.

Fig. 11 shows the measured and calculated results of the static torque. An average value of the measured torque is 1.87 $\mathrm{Nm}$. There is an error of $1.32 \%$ between the measured and calculated torques. The torque ripple is originated due to cogging torque. However, the ripple may not influence the flow rate of the centrifugal pump.

\section{ROTATIONAL CHARACTERISTICS}

In this section, rotational characteristics are investigated experimentally. Fig. 12 shows the rotational characteristics of the magnetically suspended shaft. Fig. 12(a) shows the vibration amplitudes against the rotational speed. The measurements are carried out every $300 \mathrm{r} / \mathrm{min}$ until 6,000 $\mathrm{r} / \mathrm{min}$. The test machine is possible to be driven up to the speed of $6,000 \mathrm{r} / \mathrm{min}$. The maximum peak is observed at a rotational speed of $300 \mathrm{r} / \mathrm{min}$. It may be caused due to the rotor dynamics. The detail about the rotor dynamics is under investigation and will be presented in future works. The maximum vibration amplitude is about $x=0.11 \mathrm{~mm}, y=0.14$ $\mathrm{mm}, \theta_{x}=1.21 \mathrm{mrad}, \theta_{y}=1.05 \mathrm{mrad}$ at $300 \mathrm{r} / \mathrm{min}$, which is less than one third of the touchdown clearance. It is seen that the vibration amplitudes are relatively small compared to movable limitations in the radial and tilting directions.

Figures 12(b) and 12(c) show the radial and tilting positions

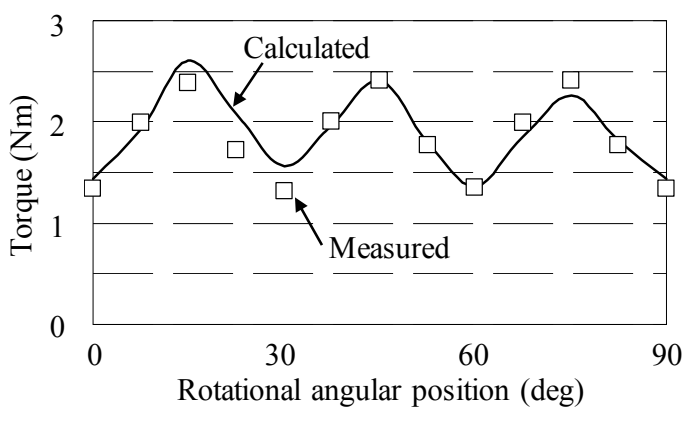

Fig. 11. Measured static torque of the bearingless PM motor with the q-axis current of $13.8 \mathrm{~A}$

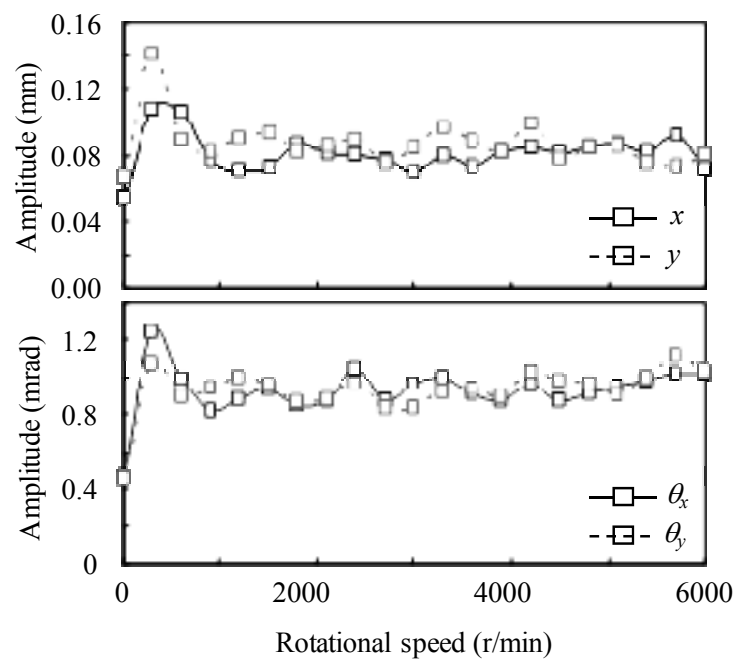

(a) Vibration amplitudes of rotor in radial and tilting direction.

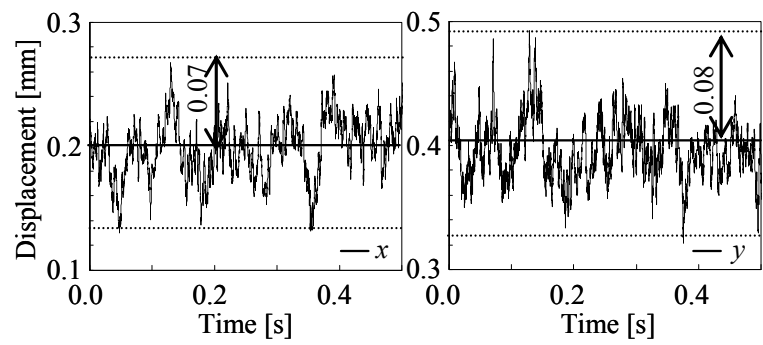

(b) Waveforms of rotor positions $x$ and $y$ in radial direction at 6,000 $\mathrm{r} / \mathrm{min}$.

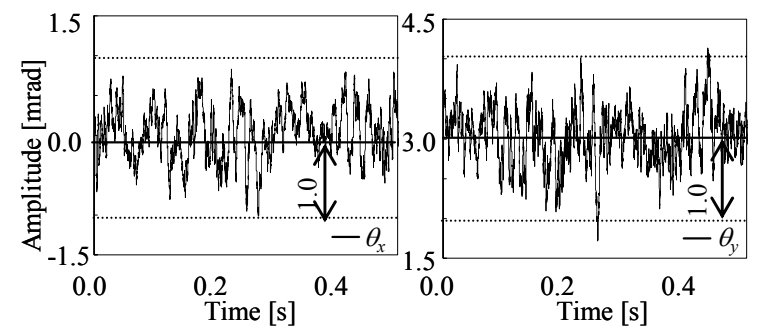

(c) Waveforms of rotor positions $\theta_{x}$ and $\theta_{y}$ in tilting direction at 6,000 $\mathrm{r} / \mathrm{min}$.

Fig. 12. Rotational characteristics of the bearingless PM motor. 
at $6,000 \mathrm{r} / \mathrm{min}$. These figures show the stable rotation at speeds up to $6,000 \mathrm{r} / \mathrm{min}$. The specification of rotation test up to $5,500 \mathrm{r} / \mathrm{min}$ is satisfied.

\section{CONCLUSIONS}

A new 5-axis actively controlled bearingless drive system is developed for centrifugal pumps. The drive system includes two consequent-pole type bearingless PM motor units and a thrust magnetic bearing. The specified suspension forces of $100 \mathrm{~N}$ and $110 \mathrm{~N}$ in radial and thrust directions, respectively are successfully met. Moreover, it is shown that the test machine can be driven up to $6,000 \mathrm{r} / \mathrm{min}$ with non contact magnetic suspension. The design specifications are experimentally evaluated for stable operation.

\section{REFERENCES}

[1] A. Chiba, T. Fukao, O. Ichikawa, M. Oshima, M. Takemoto, and D. G Dorrell "Magnetic Bearings and Bearingless Drives", Elsevier, The Netherlands :2005 Mar. 381pages ISBN 0750657278

[2] H. Zhu and L. Fang, "Suspension Principle and Digital Control for Bearingless Permanent Magnet Slice Motors", Power Electronics and Motion Control Conference, 2006. IPEMC 2006. CES/IEEE 5th International, Shanghai, China, Aug. 2006.

[3] T. Nussbaumer, K. Raggl, P. Boesch, and K.W. Kolar, "Trends in Integration for Magnetically Levitated Pump Systems", Power Conversion Conference - Nagoya, 2007. PCC '07, Nagoya, Japan, Apr. 2007.

[4] H. Kurosu, T. Masuzawa, A. Katoh, K. Suzuki, H. Onuma and K. Kakihara, "Wide Gap Magnetic Bearing System For A Magnetically Suspended Clean Pump", Proc. Seventh International Symp. on Magnetic Bearings, Nara, Japan, Aug. 2008

[5] D.J. Farrar, K. Bourque, C.P. Dague, C.J. Cotter, and V.L. Poirier, "Design Feature, Development Status, and Experimental Results with the Heartmate III Centrifugal Left Ventricular Assist System with a Magnetically Levitated Rotor, ASAIO Journal 2007, Vol. 53, pp. 310$315,2007$.

[6] J. Amemiya, A. Chiba, D. G. Dorrell and T. Fukao "Basic Characteristics of a Consequent-Pole-Type Bearingless Motor" IEEE Trans. Magnetics, vol.41 no.1 pp.82-89, Jan. 2005.

[7] M. Oshima, S. Miyazawa, T. Deido, A. Chiba, F. Nakamura and T. Fukao "Characteristics of a Permanent Magnet Type Bearingless Motor" IEEE Trans. Industry applications, vol.32, No.2, pp.363-370, Apr. 1996.

[8] K. Asami, A. Chiba, M. A. Rahman, T. Hoshino and A. Nakajima "Stiffness Analysis of a Magnetically Suspended Bearingless Motor With Permanent Magnet Passive Positioning" IEEE Trans. Industry Applications vol.41, no.10, pp.3820-3822, Oct. 2005.

[9] G. Genta, L. Mazzocchetti, and E. Rava "Magnetic Suspension for a Turbomolecular Pump" in Proc. 2nd Int. Symp. Magnetic Bearings, pp.65-72, 1990.

[10] M. Antila, E. Lantto, J. Sarri, H. Esa, O. Lindgren, and K. Saily "Design of Water Treatment Compressors Equipped with Active Magnetic Bearings", in Proc. 5th Int. Symp. Magnetic Bearings, pp.389-394, 2006

[11] R. Schöb, N. Barletta and J. Hahn "The Bearingless Centrifugal Pump A Perfect Example Of A Mechatronics System", $1^{\text {st }}$ IFAC-Conference on Mechatronic System, Sep. 2000

[12] M. Amada, N. Tanabe, J. Asama, A. Chiba, S. Iwasaki, M. Takemoto, T Fukao and M. A. Rahman "Suspension Characteristics of a ConsequentPole Type Bearingless PM Motor with Wide Magnetic Gaps", IEEE Power and Energy Society Annual General Meeting - Conversion and Delivery of Electrical Energy in the 21st Century, Pittsburgh, PA, USA, July 2008. 


\section{VIII.BIOGRAPHIES}

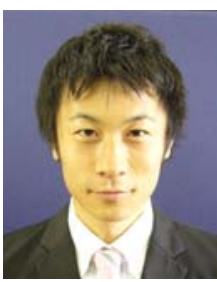

Junichi Asama (M'08) was born in Niigata, Japan, in 1979. He received the B.S., M.S. and Ph.D. degrees in Mechanical Engineering from the Tokyo Institute of Technology, in 2002, 2004 and 2006, respectively. In 2006, he was a postdoctoral researcher in the Precision and Intelligence Laboratory, Tokyo Institute of Technology. In 2007, he joined the Tokyo University of Science as a Research Associate in the Department of Electrical Engineering in the Faculty of Science and Technology. In 2009, he joined the Shizuoka University, Hamamatsu, Japan, as an Associate Professor in the Department of Mechanical Engineering in the Faculty of Engineering. He is engaged in research on bearingless motor drive systems and their applications. He is a member of the Institute of Electrical Engineers of Japan (IEEJ) and Japan Society for Precision Engineering (JSPE) in Japan.

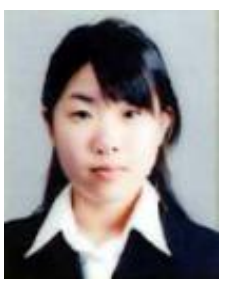

Miya Amada was born on October 11, 1984 in Tokyo, Japan. She received the B.S degree in Electrical Engineering from the Tokyo University of Science, in 2007. She is currently working toward the M.S degree. She is engaged in research on bearingless motors and their applications. She is a student member of the Institute of Electrical and Electronics Engineers.

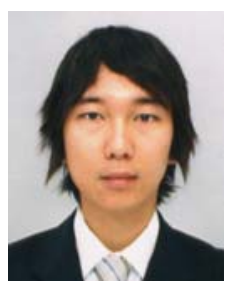

Norimasa Tanabe was born on June 13, 1982 in Saitama prefecture, Japan. He received the B.S degree in Electrical Engineering from the Tokyo University of Science, in 2006 . He is currently working toward the M.S degree. He is engaged in research on bearingless motor drive systems and their applications.

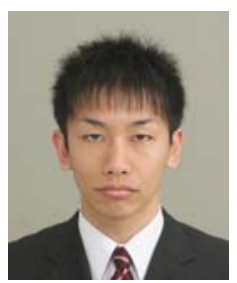

Naoya Miyamoto was born on December 26, 1985 in Ibaraki, Japan. He received the B.S degree in Electrical Engineering from the Tokyo University of Science, in 2008. He is currently working toward the M.S degree. $\mathrm{He}$ is engaged in research on bearingless motors and their applications.

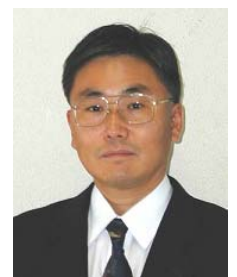

Akira Chiba (S'82-M'88-SM'97-F'07) was born in Tokyo, Japan in 1960. He received the BS., MS. and $\mathrm{Ph} . \mathrm{D}$. degrees in Electrical Engineering from the Tokyo Institute of Technology, in 1983, 1985 and 1988, respectively. In 1988, he joined the Tokyo University of Science as a Research Associate in the Department of Electrical Engineering in the Faculty of Science and Technology. From 1992 to 1993 and from 1993 to 1997 he was Research Lecturer and Senior Lecturer, respectively. He became an Associate Professor in 1997 and now a ful Professor since 2004. In 1990-91, he was an NSERC (Natural Science and Engineering Research Council of Canada) International Post-Doctoral Fellow in the Memorial University of Newfoundland, Canada. He has been studying magnetically suspended bearingless ac motors and super high-speed drives. He has so far published more than 375 papers including the first book on "Magnetic bearings and bearingless drives" in 2005, and holds 46 patents. He is a member of IEEJ in Japan and Fellow, IEEE.

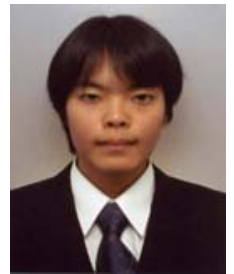

Satoru Iwasaki was born in Tokyo, Japan, in 1985. He received the B.S. degree in Mechanical Systems Engineering from the Musashi Institute of Technology, Tokyo, Japan, in 2007. Since 2007, he has been studying toward the M.S. degree at the Musashi Institute of Technology. He is engaged in research on bearingless motors and their applications. Mr. Iwasaki is a student member of the Institute of Electrical Engineers of Japan.

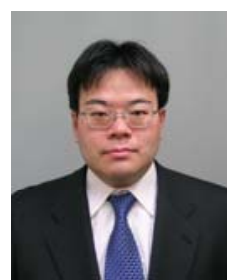

Masatsugu Takemoto (M'99) was born in Tokyo, Japan, in 1972. He received the B.S. and M.S. degrees in electrical engineering from the Tokyo University of Science, in 1997 and 1999, respectively. In 2005, he received the Ph.D. degree in electrical engineering from the Tokyo Institute of Technology. In 1999, he joined the Tokyo Institute of Technology, Tokyo, Japan, as a Research Associate in the Department of Electrical Engineering. In 2004, he joined the Musashi Institute of Technology, Tokyo, Japan, as a Research Associate in the Department of Mechanical Systems Engineering, where he became a Lecturer in 2005. In 2008, he joined the Hokkaido University, Hokkaido, Japan, where he is an Associate Professor with Division of Systems Science and Informatics. He is engaged in research on bearingless motor drive systems and permanent magnet synchronous motors. Dr. Takemoto is a member of the Institute of Electrical Engineers of Japan.

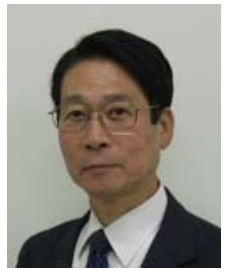

Tadashi Fukao (M'85-SM'93-F'94) was born in Shizuoka Prefecture, Japan in 1940 . He received the BS., MS. and Ph.D. degrees in Electrical Engineering from the Tokyo Institute of Technology, in 1964, 1966 and 1969, respectively. In 1968 he joined the Tokyo Institute of Technology as a Research Associate in the Department of Electrical and Electronic Engineering. From 1977-1986 and from 1986-2001 he was an Associate Professor and Professor, respectively. In 2001 he joined the Musashi Institute of Technology. He has been a Professor with Department of Mechanical Systems Engineering. He is engaged in research on high speed motors and bearingless motor drive systems. He was the president of the Institute of Electrical Engineers of Japan from 2003 to 2004. He received the IEEE William E. Newell Technical Field Award in 2009.

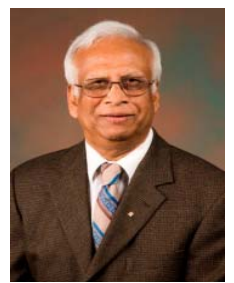

M. Azizur Rahman (S'66-M'68-SM'73-F'88, LF 2007) was born at Santahar, Bangladesh on January 9 , 1941. He received the B. Sc. Eng. from Bangladesh University of Engineering and Technology (BUET), Dhaka, the M. A. Sc. from the University of Toronto, Canada, and the $\mathrm{PhD}$. degrees from Carleton University, Ottawa, Canada all in Electrical Engineering in 1962, 1965 and 1968, respectively, In 1962, he joined BUET as a Lecturer and was promoted to Full Professor in 1975. In 1976, he joined the Memorial University of Newfoundland, Canada, where is a Professor and University Research Professor. He has 47 years of teaching including about 10 years of full-time and concurrent industrial, utility and consulting experiences at GE, Schenectady, GE Canada, Peterborough, Newfoundland Hydro, Dhaka Electric Supply, Teshmont Consultants, Iron Ore Company of Canada, etc. He has been a Visiting Professor and Research Fellow at the Imperial College, London, Technical University of Eindhoven, University of Manitoba and University of Toronto, Nanyang Technological University, Tokyo Institute of Technology, University of Hong Kong, Tokyo University of Science and University of Malaya. He has published more than 635 papers including eleven patents, and two books as well as 5 book chapters. His current research interests are in machines, intelligent controls, power systems, digital protection and power electronics and wireless communications. He has been bestowed upon with numerous awards including the GE Centennial Invention Disclosure Award in 1978, IEEE Outstanding Students Counselor's Award in 1980, IEEE Notable Service Award for contributions to IEEE and Engineering Professions in 1987, IEEE Industry Application Society's Outstanding 
Achievement Award in 1992, Association of Professional Engineers and Geoscientists of Newfoundland Merit Award in 1994, IEEE Canada Outstanding Engineering Educator's Medal in 1996, IEEE Third Millennium Medal 2000, IEEE Cyril Veinott Electromechanical Energy Conversion Award in 2003, IEEE William E. Newell Power Electronics Award in 2004, Khwarizmi International Award in 2005, IEEE Dr. -Ing. Eugine Mittelmann Achievement Award in 2007 and the IEEE Richard H. Kaufmann Technical Field Award in 2007, the A. D. Dunton Award of Distinction and the IEEEPES Distinguished Service Award in 2008. He is a registered Professional Engineer in the Province of Newfoundland and Labrador, Canada, a member of the Institution of Electrical Engineers, Japan, a Fellow of the Institution of Engineering and Technology, U.K., a Fellow of the Engineering Institute of Canada and a Life Fellow of the Institution of Engineers, Bangladesh and a Fellow of the Canadian Academy of Engineering. 